\title{
Discrepancy between regional left ventricular regional circumferential strain assessed by MR-tagging and by speckle tracking echocardiography
}

\author{
S. El Ghannudi ${ }^{1,3}$, P. Germain 1, ${ }^{2}$, MY J eung ${ }^{1}$, H Samet ${ }^{2}$, A Trihn ${ }^{2}$, H Petit-Eisenmann ${ }^{2}$, E Durand ${ }^{3}$, \\ C Roy ${ }^{1}$, A Gangi ${ }^{1}$ \\ 1. Department of Radiology, University Hospital, Strasbourg, France. 2. Department of Cardiology, University Hospital, \\ Strasbourg, France. 3. Department of Nuclear Medicine, University Hospital, Strasbourg, France
}

Correspondence: Germain Philippe. Address: Service de Radiologie, Nouvel Hôpital Civil, 1 place de I'Hopital, 67091, Strasbourg Cedex, France. Email: germain.philippe7@gmail.com

Received: May 9, 2013

Accepted: July 16, 2013

Online Published: July 30, 2013

DOI : $10.5430 / j b g c . v 3 n 4 p 75$

URL: http://dx.doi.org/10.5430/jbgc.v3n4p75

\section{Abstract}

Background: In recent years, myocardial strain imaging has gained an important place for the evaluation of cardiac patients. Global longitudinal strain assessed by speckle tracking echocardiography (STE) is now commonly used but circumferential strain remains less extensively studied. MR-tagging is recognized as the reference method for circumferential strain analysis, however no validation study between regional MR-tagging and regional STE has been performed up to now.

Objective: To compare segmental circumferential strain values (Ecc) obtained by speckle tracking and by MR-tagging in patient with normal systolic function in order to define if both methods are interchangeable or not.

Patients and methods: patients without significant regional nor global systolic dysfunction (LVEF > 55\%) were studied by MR-tagging ( $n=82$ ) and by STE $(n=35)$. Left ventricular mid-level short axis slice was obtained by both methods and paired data were available in 16 patients. Segmental Ecc values were computed in six equidistant sectors using GE EchoPac software for STE and InTag post processing software for MR-tagging.

Results: 1) Comparison between regions: Overall results showed that regional peak Ecc magnitude |Eccpeak| was not uniform with both methods but in an opposite way. MR-tagging demonstrated significantly lower septal |Eccpeak| as compared with postero-lateral |Eccpeak| (-16.5 \pm 3.6 vs $\left.-23.4 \pm 4.4, p<10^{-4}\right)$. Conversely, STE showed significantly higher septal |Eccpeak| as compared with postero-lateral |Eccpeak| (-22.3 $\left.\pm 6.4 v s-13.9 \pm 6.2, p<10^{-4}\right)$. 2) Comparison between both methods: In the subgroup of patients studied by both methods, septal |Eccpeak| was $29 \%$ lower by MR-tagging as compared with STE $(-14.9 \pm 2.4$ vs $-20.9 \pm 6.5, p<.006)$ and postero-lateral |Eccpeak| was $39 \%$ lower by STE as compared with MR-tagging $(-12.9 \pm 5.9$ vs $-21.0 \pm 2.9, p<.0003)$. 3) Intra and interobserver coefficients of variation were homogeneous (in the range 10\%-14\%) for all sectors with MR-tagging but were dramatically variable with STE (15\% to $20 \%$ in the anterior-septal region but three times higher, in the range $35 \%-40 \%$, in the postero-lateral territory).

Conclusion: Regional distributions of Ecc is not uniform but opposite results are provided by MR-tagging and by STE. This finding demonstrates that both methods cannot be considered as interchangeable. These conflicting results raise the 
question of the validity of either MR tagging or speckle tracking for the quantification of regional circumferential strain. Some arguments, developed in the discussion would rather let believe that MR-tagging results should be more reliable.

\section{Key words}

Magnetic resonance tagging, Speckle tracking echocardiography, Regional systolic function, Circumferential strain, Left ventricle

\section{I ntroduction}

Analysis of regional left ventricular (LV) contraction is important to establish the consequences of myocardial dysfunction, especially in ischemic heart disease. Measurement of myocardial deformations quantified by strain provides a more detailed analysis of regional contraction than the simple visual analysis of the endocardial contour excursion or segmental wall thickening (i.e. ASE wall motion score). Strain is insensitive to passive displacements (tethering effect) but is specific of active movements and myocardial tagging has demonstrated improved ability to detect dobutamine-induced wall motion abnormalities as compared with dobutamine-cardiac-MR without tagging ${ }^{[1]}$. Tagging-MRI (and allied techniques such as DENSE, SENC or Feature tracking) and speckle tracking echocardiography (STE) are the two main imaging modalities allowing assessment of myocardial strain. Among the three types of myocardial strain: longitudinal, radial and circumferential, the longitudinal component is now widely used and well validated by STE whereas the circumferential component (Ecc), which is the easiest to study by tagging-MR ${ }^{[2]}$ has been less extensively studied by STE. Normal values of regional Ecc, which determine the threshold for detection of segmental contraction abnormalities, appear to be quite variable in the literature (e.g. higher septal Ecc values by STE ${ }^{[3,4]}$ and lower septal Ecc values by MR-tagging ${ }^{[5]}$ as compared with lateral Ecc values). However, no direct comparative study of regional circumferential strain has been published yet between MR-tagging and STE. For this reason, we thought to compare the results obtained with these two methods in order to confirm or refute regional discrepancies between both techniques. A corollary to this question was to determine whether it would be possible to transpose MR regional metrics to STE measurements in clinical studies requiring a large number of observations.

\section{Materials and methods}

\subsection{Study population}

Eighty two patients were studied by MR-tagging and 35 patients were studied by STE. Paired data were available in 16 patients, in whom both examinations were performed within a few days apart ( $<$ three days). The MR-tagging group $(n=82)$ was composed by 18 normal volunteers from our cardiology and radiology team and by 64 patients. The reason for MR exam in patients was atypical chest pain ( $n=13$, all demonstrating negative late enhancement imaging), myocarditis with preserved systolic function $(n=8)$, dilated aortic root $(n=17$, without significant aortic insufficiency), search for cardiac involvement in systemic disease $(n=10)$, relatives of patients with cardiomyopathy $(n=5)$ and paroxysmal rhythm disturbance, malaise or syncope $(n=11)$. Twenty five of the STE patients presented with atypical chest pain or dilated aortic root and 10 presented with myocarditis (eight of them being also included in the MR-tagging group). All patients were in normal sinus rhythm without hypertension, diabetes, ischemic myocardial lesion, valvular disease, cardiomyopathy or congestive heart disease. Systolic function was normal with left ventricular ejection fraction (LVEF) > 55\% and absence of any segmental wall abnormality at both echocardiographic and MR examinations. This observational study was performed in accordance with ethics rules of our institution and an informed consent was obtained for every patient.

\subsection{Cardiac MRI tagging acquisition}

All MRI acquisitions were performed on a 1.5T MR scanner (1.5T Espree VB15 release, Siemens Medical Solution, Erlangen Germany). From vertical and horizontal long axis planes obtained with steady-state free precession (SSFP) cine 
sequences, a mid-ventricular short axis slice was acquired with the tagging sequences (at the level of the papillary muscle). Segmented SSFP tagging cine sequences used 108 phase encoding steps, TE $=1.36 \mathrm{~ms}$, TR (temporal resolution) $=29.5$ $\mathrm{ms}$, flip angle $=20^{\circ}$, FOV $284 \mathrm{~mm}$ (in plane resolution $=1.3 \mathrm{~mm} \times 2.6 \mathrm{~mm}$ ), slice thickness $=8 \mathrm{~mm}$. A tagging grid pattern using spatial modulation of the magnetization (CSPAMM) was applied with intertag distance set to $6 \mathrm{~mm}$. Twenty to 36 phases were gathered according to the patient's heart rate. Additionally, LV volumetry was obtained from a stack of non tagged short axis slices encompassing the LV.

\subsection{Tagging data post-processing}

MR tagged images were processed off-line with Osirix software, using the Intag Plugin (www.creatis.insa-lyon.fr/inTag/) developed by Arts et $a l^{[6]}$. After semi-automated endocardial and epicardial contour tracing and manual positioning of the right ventricular connection to the upper septum (see Figure 1A), the short axis slice was divided in 6 polar sectors (anteroseptal, inferoseptal, inferior, posterior, lateral and anterior). Ecc data points were exported as XLS files (Microsoft Excel format) and processed by a custom program written in Microsoft Visual C. In order to remove noise and to reduce outliers, Ecc curves were smoothed by a second order Fourier filter. Peak Ecc and time-to-peak were extracted. Moreover in order to visualize contraction non-uniformity, regional variance vector (RVV), described by Helm et al ${ }^{[7]}$ was determined by summing end-systolic strain vectors at each sector points. If all vectors have the same magnitude (homogeneous contraction) the corresponding vectorial sum will be null. Conversely, clustered regional inhomogeneity will lead to greater RVV.

Figure 1. A) From the mid-level short axis tagged images (left), the myocardium was divided in 6 sectors and the corresponding regional circumferential strain curves were extracted by the InTag software (right). Greatest |Eccpeak $\mid$ magnitudes were seen in the lateral territory and smallest |Eccpeak| were seen in the septal territory. B) A similar process was performed from echocardiographic frames (left) with the EchoPac software. Circumferential strain curves (right) showed greatest $\mid$ Eccpeak $\mid$ magnitudes in the septal territory and smallest |Eccpeak $\mid$ in the posterolateral region.

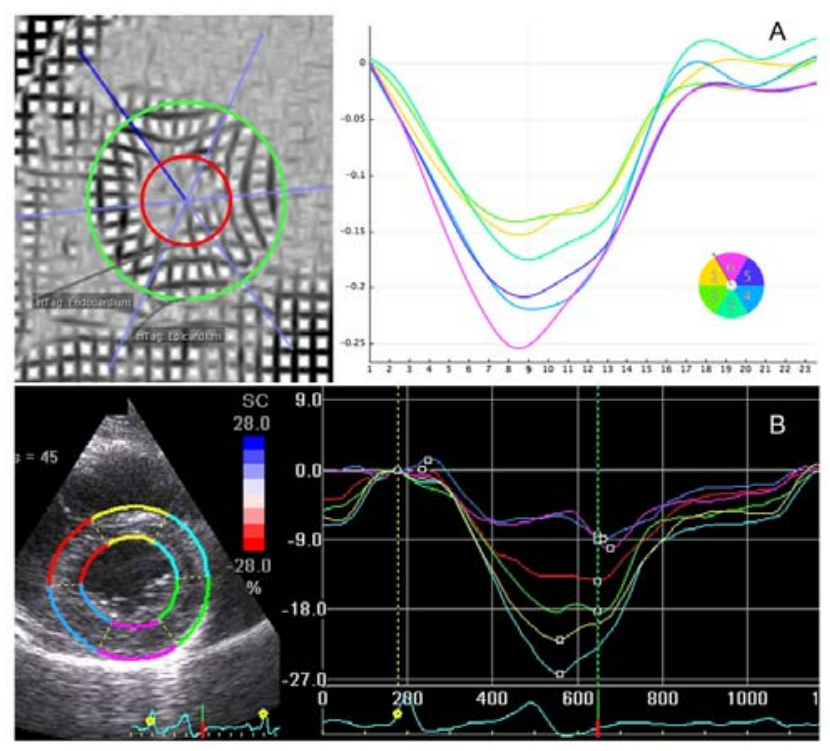

\subsection{Speckle tracking strain analysis by echocardiography}

Echocardiographic examination was performed according to the ESC guideline ${ }^{[8]}$ using a General Electric Vivid 7 (GE Healthcare) with a 1.7/3.4 (second harmonic) MHz echo transducer. High frame rate $(>66 \mathrm{~Hz}$ ) midventricular short axis slice (at the level of papillary muscles) was obtained for strain analysis with cross sectional image made as circular as possible. EchoPac 2D acoustic-tracking software (V7.2.0) was used to extract regional Ecc in the six equidistant standard sectors (as illustrated in Figure 1B). The entire myocardial thickness was included in the region of interest, taking care to avoid pericardium. The adequacy of tracking was optimized manually and the tracking score was approved by the software for all segments. Echo readers (SE and HS) and MR readers (PG and MYJ) were trained senior cardiologist or radiologist, blinded to the results obtained by each other. 


\subsection{Statistical analysis}

Continuous variables are expressed as mean \pm standard deviation (SD). The mean values of each index were compared with one-way analysis of variance and post hoc analysis was then performed using Student $t$-test. Intraobserver variability and interobserver variability were assessed in all of the 16 paired observations several months after the initial analysis, using the Bland and Altman method and the coefficient of variation ( $C V=100 \times S D / m e a n$ of paired data). Statistical analysis was performed with MedCalc12.2.1.0 software (Mariakerke, Belgium).

\section{Results}

\subsection{Global Ecc}

Subject's characteristics are listed in Table 1. Global |Eccpeak| was significantly higher with MR-tagging than with STE $(-20.2 \pm 2.4$ vs $-18.3 \pm 4.1, p<0.001)$. Despite no significant change in LVEF, smaller values of global |Eccpeak| were found with both methods in the subgroup of paired patients, because a significant number of these patients presented with myocarditis in this subgroup. Poor quality STE strain curves were frequently observed in the posterior (11/32) and lateral (6/32) sectors (i.e. abnormally oscillating curves with low amplitude, multiple peaks and even inconsistent positive stretching components).

Table 1. Subject's characteristics

\begin{tabular}{llll}
\hline Item & MR-tagging group & STE group & Paired study group \\
\hline N (female) & $82(37)$ & $35(11)$ & $16(4)$ \\
Age & $42.4 \pm 15.9$ & $44.5 \pm 16.6$ & $37.7 \pm 13.7$ \\
LVEF (\%) & $66.0 \pm 4.8$ & $63.5 \pm 6.5$ & $66.1 \pm 8.4 \mathrm{MR} / 61.6 \pm 7.4 \mathrm{echo}$ \\
Myocarditis & $8 / 82$ & $10 / 35$ & $8 / 16$ \\
Global peak Ecc (\%) & $-20.2 \pm 2.4$ & $-18.3 \pm 4.1 *$ & $-18.7 \pm 3.4 \mathrm{MR} * /-17.0 \pm 4.4$ echo * \\
\hline
\end{tabular}

Note. N: number of patients, LVEF: left ventricular ejection fraction. * $p<0.01$ vs MR-tagging group

\subsection{Comparison between regions}

Overall sectorial analysis, summarized in Table 2, showed that regional peak Ecc magnitude |Eccpeak| was not uniform with both methods but in an opposite way. As shown by Figure 2, MR-tagging demonstrated significantly lower septal |Eccpeak| as compared with postero-lateral |Eccpeak| (-16.5 \pm 3.6 vs $\left.-23.4 \pm 4.4, p<10^{-4}\right)$. Conversely, STE showed significantly higher septal |Eccpeak| as compared with postero-lateral |Eccpeak| (-22.3 \pm 6.4 vs $\left.-13.9 \pm 6.2, p<10^{-4}\right)$.

Figure 2. Diagram of regional variance vector obtained by MR-tagging from the 82 patients in the mid-level short axis slice, showing the asymmetric warping of the contraction with net vector (red arrow) oriented toward the septum due to stronger lateral circumferential strain.

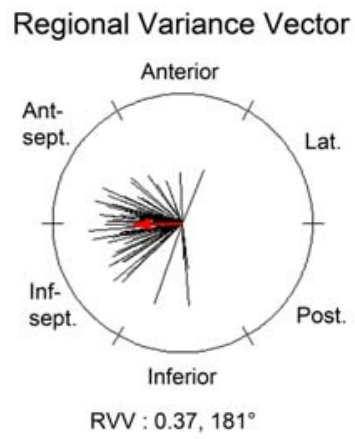


Table 2. Peak circumferential strain according to sector

\begin{tabular}{llllllllll}
\hline Ecc (\%) & $\mathbf{n}$ & Ant-sept & Inf-sept & Inferior & Posterior & Lateral & Anterior & $\boldsymbol{p}$ & Mean \\
\hline STE & 82 & $-24.2 \pm 6.3$ & $-20.4 \pm 6.6$ & $-14.9 \pm 5.6$ & $-12.0 \pm 5.9$ & $-15.7 \pm 6.6$ & $-22.5 \pm 7.3$ & $10^{-3}$ & $-18.3 \pm 4.1$ \\
TAG & 35 & $-17.1 \pm 4.2$ & $-15.9 \pm 3.0$ & $-18.8 \pm 3.8$ & $-24.2 \pm 4.1$ & $-22.7 \pm 4.7$ & $-22.3 \pm 4.5$ & $10^{-3}$ & $-20.2 \pm 2.4$ \\
$p$ & & $<10^{-3}$ & $<10^{-3}$ & $<10^{-3}$ & $<10^{-3}$ & $<10^{-3}$ & ns & 0.002 \\
\hline
\end{tabular}

Note. STE: speckle tracking data, TAG: tagging data of peak circumferential strain Ecc.

\subsection{Comparison between both methods}

In the subgroup of paired patients studied by both methods, illustrated in Figures 3 and 4, septal |Eccpeak| was 29\% lower by MR-tagging as compared with STE $(-14.9 \pm 2.4$ vs $-20.9 \pm 6.5, p<.006)$ and postero-lateral |Eccpeak| was 39\% lower by STE as compared with MR-tagging (-12.9 \pm 5.9 vs $-21.0 \pm 2.9, p<.0003)$.

Figure 3. Diagram of speckle tracking (STE) vs MR-tagging regional values of Ecc obtained in 16 patients with normal systolic function, showing significantly greater Ecc magnitude in the septal sectors by STE as compared with MR, whereas opposite results are observed in the posterolateral wall. Septal $\left|\mathrm{Ecc}_{\mathrm{peak}}\right|$ was $29 \%$ lower by MR-tagging as compared with STE $(p<.006)$ and postero-lateral $\left|\mathrm{Ecc}_{\text {peak }}\right|$ was $39 \%$ lower by STE as compared with MR-tagging $(p<.0003)$.

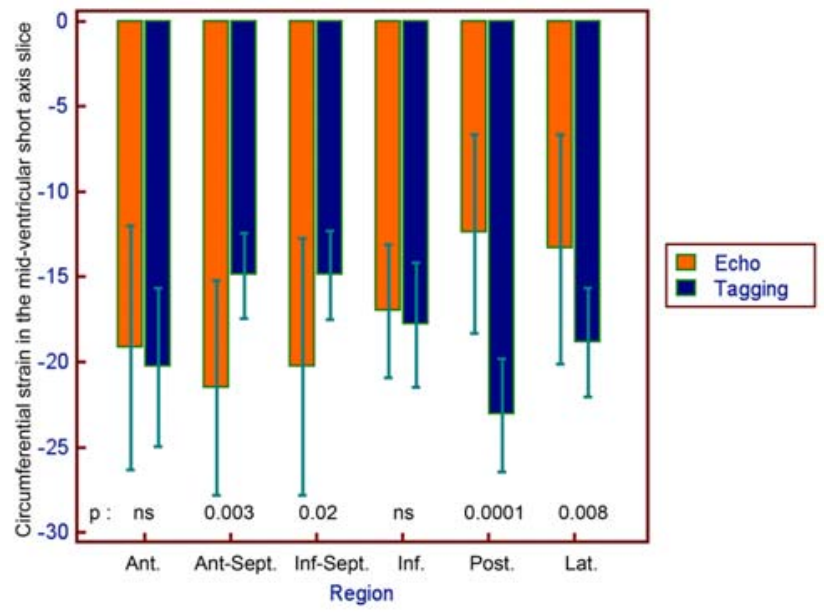

Figure 4. Bland and Altman plot of the differences between paired speckle-based (Echo) vs taggingbased (MR) segmental strain values (Ecc).

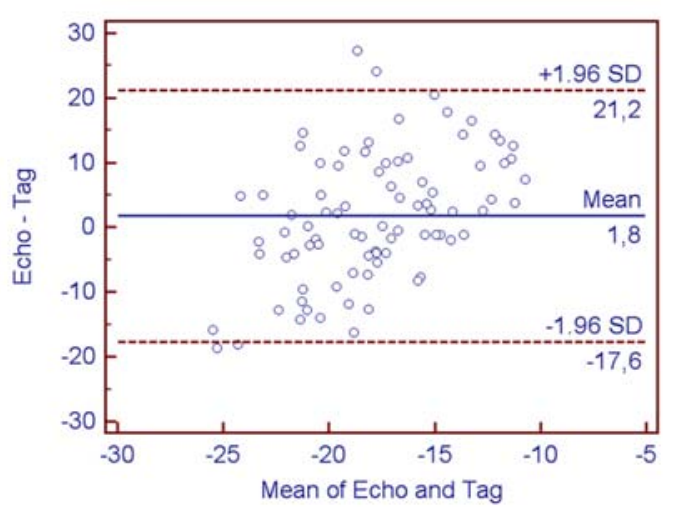

Intra and interobserver coefficients of variation, listed in Table 3, were homogeneous (in the range 10\%-14\%) for all sectors with MR-tagging but were dramatically variable with STE (15\% to 20\% in the anterior-septal region but three times higher, in the range $35 \%-40 \%$, in the postero-lateral territory).

Table 3. Intra-observer and interobserver variability for regional circumferential strain

\begin{tabular}{|c|c|c|c|c|c|c|c|}
\hline CV (\%) & & Ant-sept & Inf-sept & Inferior & Posterior & Lateral & Anterior \\
\hline STE & Intraobserver & 14.5 & 19.4 & 27.2 & 38.7 & 34.7 & 17.4 \\
\hline TAG & Intraobserver & 11.5 & 11.1 & 11.4 & 7.7 & 9.9 & 10.4 \\
\hline STE & Interobserver & 16.1 & 19.0 & 23.7 & 43.1 & 41.6 & 17.1 \\
\hline TAG & Interobserver & 13.2 & 13.0 & 14.1 & 8.5 & 11.0 & 11.2 \\
\hline
\end{tabular}

Note. Intra and interobserver coefficient of variation (\%). STE: speckle tracking data, TAG: tagging data of peak circumferential strain Ecc. 


\section{Discussion}

Cardiac contraction is a complex phenomenon, explained by myocardial fibers architecture and resulting in three main deformation components: longitudinal, circumferential (Ecc) and radial. Ecc is an important determinant of contraction because almost $80 \%$ of the myofibers are oriented circumferentially ${ }^{[9]}$. However, this parameter has been much less studied by STE as compared with longitudinal strain and the question arises as to the transposition of MR results to STE. In this study we demonstrate that in patients with normal systolic function, regional non uniformity of Ecc shows a surprising opposite pattern between STE and tagging-MR (i.e. highest |Eccpeak| in the septal territory by STE and highest |Eccpeak| in the posterolateral wall by MR-tagging). Paired comparison between both methods confirms these discrepancies. Thus, our finding is provocative because both MR and STE claim to provide the same Ecc metrics but their results are inconsistent; yet the truth must lie on the side of one or the other of these two methods. Now, what are the arguments that can help us solve this problem?

\subsection{Previously reported data about regional Ecc obtained by MR-tagging and by STE}

Our finding is actually consistent with published data from the literature. This discrepancy was already noticed by Hurlburt et al ${ }^{[10]}$ in adults and by Sato et al in children ${ }^{[11]}$. However, in previously published papers, regional Ecc analysis was only investigated through single modality studies (STE or MR-tagging being performed separately, without paired comparisons) and no direct comparison has been published yet about regional circumferential strain.

Few direct comparisons have been published between STE and MR-tagging based strain measurements. Overall good correlations were found for longitudinal and radial strain in healthy volunteers and in patients with ischemic heart disease ${ }^{[12-15]}$. Circumferential strain was considered by the team of Brisbane ${ }^{[14,15]}$, providing a modest correlation coefficient between both methods, between 0.51 and 0.64 . However, only overall results of strain were compared in these studies, without focusing on regional analysis. Moreover it should be emphasized that concordance between both methods was not very strong. For example, despite a good correlation coefficient ( $r=0.87)$, it should be noticed that in the study of Amundsen et al ${ }^{[13]}$ as well as in the study of Cho et al ${ }^{[14]}$, Bland-Altmann 95\% limits of agreement between STE and tagging was in the range $\pm 10 \%$, which is almost half of the mean magnitude of circumferential strain (mean value in the range -20\%). In the study of Bansal et al ${ }^{[15]} r$ was 0.63 and the $95 \%$ limits of agreement was somewhat better, in the range $\pm 6 \%$ (i.e. approximately the third of the mean circumferential strain, which is also a relatively wide spread of values).
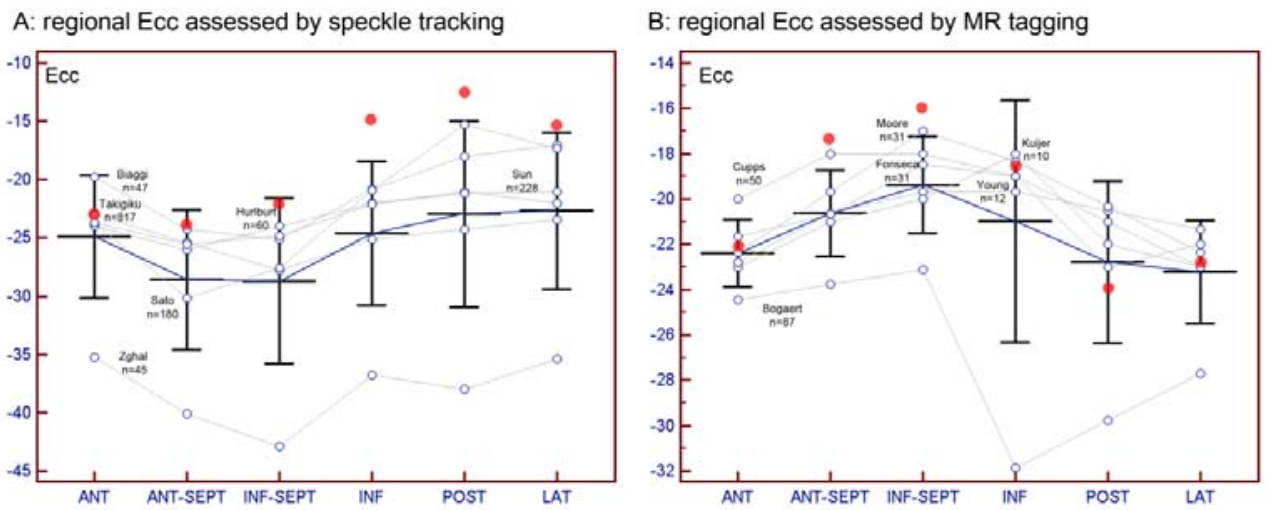

Figure 5. Comparison of published regional Ecc values obtained by speckle tracking (A) and by MR-tagging (B), showing opposite patterns (septal magnitude higher with speckle tracking whereas lateral magnitude is higher with MR-tagging). Authors (see references) and number of observations are written on the corresponding line. Some sectorial data points were interpolated if lacking in the original publication. Data from our study are presented by red dots. 
If we consider regional Ecc analysis, largely investigated through single modality studies (STE or MR-tagging performed separately), highest lateral absolute Ecc is constantly reported in MR-based literature ${ }^{[5,16-21]}$ and highest septal absolute Ecc is constantly reported in the STE based literature ${ }^{[3,4,10,11,22,23]}$. These results, are not influenced by the base-apex positioning and by the type of machine used ${ }^{[3]}$. Diagrams of the figure 5 summarize those contradictory MR and STE published results.

\subsection{Several arguments in favour of the accuracy of MR-tagging results}

Endocardial motion assessed by gated-SPECT ${ }^{[24]}$ or by radial displacement at aortic valve closure ${ }^{[25]}$ showed lowest values in the septal region and highest values in the lateral wall but these data do not necessarily imply the same result for Ecc. A stronger argument is provided by former works based on miniature rapiopaque tantalum markers implanted in several myocardial location of transplanted heart and studied by cineradiograms, showing that circumferential shortening was significantly lower in the septum than in the lateral free wall ${ }^{[26]}$.

From a physiological point of view, strain is negatively affected by wall stress as was demonstrated by Donal et al ${ }^{[27]}$ for longitudinal strain and by Hurlburt et $a l^{[10]}$ for Ecc. Systolic wall stress (SWS) relies on the level of afterload and on the ratio of wall curvature $(R)$ to wall thickness $(T)$ : the larger $R$ and the thinner $T$, the greater SWS. Geometrical analysis of segmental systolic thickness and wall curvedness of the LV led to controversial results in this issue; some studies showed significantly increased septal SWS ${ }^{[28]}$ whereas other studies did not ${ }^{[29]}$. An additional factor that might explain increased stress in the septum, not taken into account in those studies, is the possible role of the right systolic ventricular pressure, furthermore increasing septal compression.

Simple visual analysis of tag lines and rudimentary caliper measurements of intertag distance in diastole and in systole suggest that systolic septal lines narrowing in the circumferential direction is actually weaker in the septum than in the lateral wall.

\subsection{Weaknesses of echocardiography that might account for observed discrepancies}

Such basic visual assessment cannot be done by STE since pixel-block matching relies on complex signal processing exceeding capabilities of visual analysis. Speckle tracking algorithm, inherently sensitive to noise, should be less efficient in the far field (posterior and lateral walls) than in the near field (anteroseptal walls). Moreover, lateral resolution which is depth-dependent, is lower than axial resolution and this might explain shortening underestimation in the direction perpendicular to the ultrasound beam ${ }^{[13]}$. This phenomenon is related to increased speckle decorrelation in the lateral direction which does not occur similarly in the long axis direction, thus explaining that long axis strain is more reliable than circumferential strain. This assumption is supported by the fact that tracking quality is worst in the posterolateral wall ${ }^{[30]}$, intraobserver variability is higher in those segments ${ }^{[10]}$ and STE rejected data (unsuitable signal quality for reliable processing) mainly concerns the lateral or posterior walls ${ }^{[25]}$. Such was confirmed in our study as shown by the large number of poor quality strain curves in the posterior (11/32) and lateral (6/32) sectors and by the 3-fold increase of intra and interobserver variabilities observed in those far field regions. A change in the placement of the probe could theoretically help overcome this difficulty but the switch from the parasternal to the subcostal position still leaves the posterolateral wall farthest. Another deleterious phenomenon for 2D STE is the systolic out of plane motion of the speckles in short axis slices, preventing accurate motion tracking and thus strain measurement. This phenomenon does not occur with MR tagging thanks to the large through plane application of the tags. In addition, it is conceivable that stationary echoes from the pericardium might be included in the STE averaging algorithm in the inferior and lateral wall, thus leading to reduced apparent strain values.

\subsection{Study limitations}

Most of our observations were obtained from patients with normal systolic function and not exclusively from healthy volunteers. Almost half of the patients from the paired group, studied by both imaging modalities, presented with 
myocarditis. Despite preserved LVEF, significant decrease in global longitudinal strain has been demonstrated in patients with myocarditis ${ }^{[31]}$ and reduced segmental circumferential strain has also been found in the lateral wall (mostly involved by subepicardial lesions as seen by late-enhacement MR after gadolinium injection). However, in these patients, segmental strain reduction is moderate and this could not account for discrepancies observed between STE and MR-tagging. Overall Ecc values in our study were similar to those obtained in previously published data. The number of patients studied by STE is very small indeed, but so far it constitutes the sole pairwise group available for comparison between STE and MR-tagging based regional Ecc. It is unlikely that a larger effective might contradict our results, which are supported by consistent evidences from the literature. Misaligned image planes and discrepancies in myocardial contour tracing in MRI and STE might explain some of the variation. Longitudinal, radial and moreover 3D strains were not studied here owing to technical limitations of the post processing software.

\section{Conclusion}

MR-tagging based circumferential strain heterogeneity is characterized by relative septal hypocontractility whereas speckle tracking echocardiography shows opposite results with maximal strain strength in the septum. The present study, pointing this discrepancy, does not allow defining which technique is right or wrong but several theoretical arguments are in favor of the validity of MR tagging in this issue (possibly related to increased systolic wall stress in the septum and to limitations of speckle tracking algorithms in the posterior and lateral far field regions). Anyway, a practical implication is that both techniques are not interchangeable for Ecc regional analysis and that circumferential strain remains a "work in progress" still requiring several technical issues to be overcome before it might be considered as reliable as the widely recognized and clinically used longitudinal strain.

List of abbreviations: Ecc: circumferential strain, |Eccpeak| absolute value of peak Ecc, LV: left ventricular, STE: speckle tracking echocardiography, LVEF: left ventricular ejection fraction, SSFP: steady state free precession, SWS: systolic wall stress.

\section{Acknowledgements}

The authors thank Dr Joost P.A Kuijer PhD, VU University Medical Center, Amsterdam, Netherlands for developing and providing the CSPAMM tagging package (WIP), P Croisille and P Clarysse of CREATIS-LRMN, INSA Lyon, University of Lyon, Villeurbanne 69621, France for providing the Post processing InTag software and useful advices.

\section{References}

[1] Kuijpers D, Ho KY, van Dijkman PR, Vliegenthart R, Oudkerk M. Dobutamine cardiovascular magnetic resonance for the detection of myocardial ischemia with the use of myocardial tagging. Circulation. 2003; 107(12):1592-7. PMID: 12668491. http://dx.doi.org/10.1161/01.CIR.0000060544.41744.7C

[2] Jeung MY, Germain P, Croisille P, El ghannudi S, Roy C, Gangi A. Myocardial tagging with MR imaging: overview of normal and pathologic findings. Radiographics. 2012; 32(5):1381-98. PMID: 22977026. http://dx.doi.org/10.1148/rg.325115098

[3] Takigiku K, Takeuchi M, Izumi C, Yuda S, Sakata K, Ohte N, Tanabe K, Nakatani S; JUSTICE investigators. Normal range of left ventricular 2-dimensional strain: Japanese Ultrasound Speckle Tracking of the Left Ventricle (JUSTICE) study. Circ J. 2012; 76(11):2623-32. PMID: 22813873. http://dx.doi.org/10.1253/circj.CJ-12-0264

[4] Sun JP, Lee AP, Wu C, Lam YY, Hung MJ, Chen L, et al. Quantification of left ventricular regional myocardial function using two-dimensional speckle tracking echocardiography in healthy volunteers - A multi-center study. Int J Cardiol. 2012. PMID: 22365315. http://dx.doi.org/10.1016/j.ijcard.2012.01.071

[5] Moore CC, Lugo-Olivieri CH, McVeigh ER, Zerhouni EA (2000) Three-dimensional systolic strain patterns in the normal human left ventricle: characterization with tagged MR imaging. Radiology 2000; 214(2):453-466. PMID: 10671594.

[6] Arts T, Prinzen FW, Delhaas T, Milles JR, Rossi AC, Clarysse P. Mapping displacement and deformation of the heart with local sine-wave modeling. IEEE Trans Med Imaging. 2010; 29(5):1114-23. PMID: 20335094.

http://dx.doi.org/10.1109/TMI.2009.2037955 
[7] Helm RH, Leclercq C, Faris OP, Ozturk C, McVeigh E, Lardo AC, et al. Cardiac dyssynchrony analysis using circumferential versus longitudinal strain: implications for assessing cardiac resynchronization. Circulation. 2005; 111(21):2760-7. PMID: 15911694. http://dx.doi.org/10.1161/CIRCULATIONAHA.104.508457

[8] Lang RM, Bierig M, Devereux RB, Flachskampf FA, Foster E, Pellikka PA, et al. American Society of Echocardiography's Nomenclature and Standards Committee; Task Force on Chamber Quantification; American College of Cardiology Echocardiography Committee; American Heart Association; European Association of Echocardiography, European Society of Cardiology. Recommendations for chamber quantification. Eur J Echocardiogr. 2006; 7(2):79-108. PMID: 16458610. http://dx.doi.org/10.1016/j.euje.2005.12.014

[9] Streeter DD Jr, Spotnitz HM, Patel DP, Ross J Jr, Sonnenblick EH. Fiber orientation in the canine left ventricle during diastole and systole. Circ Res. 1969; 24(3):339-47. PMID: 5766515. http://dx.doi.org/10.1161/01.RES.24.3.339

[10] Hurlburt HM, Aurigemma GP, Hill JC, Narayanan A, Gaasch WH, Vinch CS, et al. Direct ultrasound measurement of longitudinal, circumferential, and radial strain using 2-dimensional strain imaging in normal adults. Echocardiography. 2007; 24(7):723-31. PMID: 17651101. http://dx.doi.org/10.1111/j.1540-8175.2007.00460.x

[11] Sato Y, Maruyama A, Ichihashi K. Myocardial strain of the left ventricle in normal children. J Cardiol. 2012; 60(2):145-9. PMID: 22483098. http://dx.doi.org/10.1016/j.jjcc.2012.01.015

[12] Edvardsen T, Gerber BL, Garot J, Bluemke DA, Lima JA, Smiseth OA. Quantitative assessment of intrinsic regional myocardial deformation by Doppler strain rate echocardiography in humans: validation against three-dimensional tagged magnetic resonance imaging. Circulation. 2002; 106(1):50-6. PMID: 12093769. http://dx.doi.org/10.1161/01.CIR.0000019907.77526.75

[13] Amundsen BH, Helle-Valle T, Edvardsen T, Torp H, Crosby J, Lyseggen E, et al. Noninvasive myocardial strain measurement by speckle tracking echocardiography: validation against sonomicrometry and tagged magnetic resonance imaging. J Am Coll Cardiol. 2006; 47(4):789-93. PMID: 16487846. http://dx.doi.org/10.1016/j.jacc.2005.10.040

[14] Cho GY, Chan J, Leano R, Strudwick M, Marwick TH. Comparison of two-dimensional speckle and tissue velocity based strain and validation with harmonic phase magnetic resonance imaging. Am J Cardiol. 2006; 97(11):1661-6. PMID: 16728234. http://dx.doi.org/10.1016/j.amjcard.2005.12.063

[15] Bansal M, Cho GY, Chan J, Leano R, Haluska BA, Marwick TH. Feasibility and accuracy of different techniques of two-dimensional speckle based strain and validation with harmonic phase magnetic resonance imaging. J Am Soc Echocardiogr. 2008; 21(12):1318-25. PMID: 19041575 http://dx.doi.org/10.1016/j.echo.2008.09.021

[16] Young AA, Kramer CM, Ferrari VA, Axel L, Reichek N. Three-dimensional left ventricular deformation in hypertrophic cardiomyopathy. Circulation. 1994; 90(2):854-67. PMID: 8044957. http://dx.doi.org/10.1161/01.CIR.90.2.854

[17] Bogaert J, Rademakers FE. Regional nonuniformity of normal adult human left ventricle. Am J Physiol Heart Circ Physiol. 2001; 280(2):H610-20. PMID: 11158958.

[18] Kuijer JP, Marcus JT, Götte MJ, van Rossum AC, Heethaar RM. Three-dimensional myocardial strains at end-systole and during diastole in the left ventricle of normal humans. J Cardiovasc Magn Reson. 2002; 4(3):341-51. PMID: 12234105.

[19] Cupps BP, Taggar AK, Reynolds LM, Lawton JS, Pasque MK. Regional myocardial contractile function: multiparametric strain mapping. Interact Cardiovasc Thorac Surg. 2010; 10(6):953-7. PMID: 20233804. http://dx.doi.org/10.1510/icvts.2009.220384

[20] Rosen BD, Edvardsen T, Lai S, Castillo E, Pan L, Jerosch-Herold M, et al. Left ventricular concentric remodeling is associated with decreased global and regional systolic function: the Multi-Ethnic Study of Atherosclerosis. Circulation. 2005; 112(7):984-91. PMID: 16103253. http://dx.doi.org/10.1161/CIRCULATIONAHA.104.500488

[21] Fonseca CG, Oxenham HC, Cowan BR, Occleshaw CJ, Young AA. Aging alters patterns of regional nonuniformity in LV strain relaxation: a 3-D MR tissue tagging study. Am J Physiol Heart Circ Physiol. 2003; 285(2):H621-30. PMID: 12689861. http://dx.doi.org/10.1152/ajpheart.01063.2002

[22] Zghal F, Bougteb H, Réant P, Lafitte S, Roudaut R. Assessing global and regional left ventricular myocardial function in elderly patients using the bidimensional strain method. Echocardiography. 2011; 28(9):978-82. PMID: 21854428. http://dx.doi.org/10.1111/j.1540-8175.2011.01476.x

[23] Biaggi P, Carasso S, Garceau P, Greutmann M, Gruner C, Tsang W, et al. Comparison of two different speckle tracking software systems: does the method matter? Echocardiography. 2011; 28(5):539-47. PMID: 21517954. http://dx.doi.org/10.1111/j.1540-8175.2011.01386.x

[24] Sharir T, Berman DS, Waechter PB, Areeda J, Kavanagh PB, Gerlach J, et al. Quantitative analysis of regional motion and thickening by gated myocardial perfusion SPECT: normal heterogeneity and criteria for abnormality. J Nucl Med. 2001; 42(11):1630-8. PMID: 11696631.

[25] Lindqvist P, Borgström E, Gustafsson U, Mörner S, Henein MY. Asynchronous normal regional left ventricular function assessed by speckle tracking echocardiography: appearances can be deceptive. Int J Cardiol. 2009; 134(2):195-200. PMID: 18508139. http://dx.doi.org/ 10.1016/j.ijcard.2008.01.045 
[26] Ingels NB Jr, Hansen DE, Daughters GT 2nd, Stinson EB, Alderman EL, Miller DC. Relation between longitudinal, circumferential, and oblique shortening and torsional deformation in the left ventricle of the transplanted human heart. Circ Res. 1989; 64(5):915-27. PMID: 2650919. http://dx.doi.org/ 10.1161/01.RES.64.5.915

[27] Donal E, Bergerot C, Thibault H, Ernande L, Loufoua J, Augeul L, et al. Influence of afterload on left ventricular radial and longitudinal systolic functions: a two-dimensional strain imaging study. Eur J Echocardiogr. 2009; 10(8):914-21. PMID: 19666722. http://dx.doi.org/10.1093/ejechocard/jep095

[28] Balzer P, Furber A, Delépine S, Rouleau F, Lethimonnier F, Morel O, et al. Regional assessment of wall curvature and wall stress in left ventricle with magnetic resonance imaging. Am J Physiol. 1999; 277(3 Pt 2):H901-10. PMID: 10484409.

[29] Shudo Y, Taniguchi K, Takeda K, Sakaguchi T, Matsue H, Izutani H, et al. Assessment of regional myocardial wall stress before and after surgical correction of functional ischaemic mitral regurgitation using multidetector computed tomography and novel software system. Eur J Cardiothorac Surg. 2010; 38(2):163-70. PMID: 20619218. http://dx.doi.org/10.1016/j.ejcts.2010.01.029

[30] Marwick TH, Leano RL, Brown J, Sun JP, Hoffmann R, Lysyansky P, et al. Myocardial strain measurement with 2-dimensional speckle-tracking echocardiography: definition of normal range. JACC Cardiovasc Imaging. 2009; 2(1):80-4. PMID: 19356538. http://dx.doi.org/10.1016/j.jcmg.2007.12.007

[31] Di Bella G, Gaeta M, Pingitore A, Oreto G, Zito C, Minutoli F, et al. Myocardial deformation in acute myocarditis with normal left ventricular wall motion--a cardiac magnetic resonance and 2-dimensional strain echocardiographic study. Circ J. 2010; 74(6):1205-13. PMID: 20453384. http://dx.doi.org/10.1253/circj.CJ-10-0017 